\title{
Estimating bolt tightness using transverse natural frequencies
}

Sah, Si Mohamed; Thomsen, Jon Juel; Brøns, Marie; Fidlin, Alexander; Tcherniak, Dmitri

Published in:

Journal of Sound and Vibration

Link to article, DOI:

10.1016/j.jsv.2018.05.040

Publication date:

2018

Document Version

Peer reviewed version

Link back to DTU Orbit

Citation (APA):

Sah, S. M., Thomsen, J. J., Brøns, M., Fidlin, A., \& Tcherniak, D. (2018). Estimating bolt tightness using transverse natural frequencies. Journal of Sound and Vibration, 431, 137-149.

https://doi.org/10.1016/j.jsv.2018.05.040

\section{General rights}

Copyright and moral rights for the publications made accessible in the public portal are retained by the authors and/or other copyright owners and it is a condition of accessing publications that users recognise and abide by the legal requirements associated with these rights.

- Users may download and print one copy of any publication from the public portal for the purpose of private study or research.

- You may not further distribute the material or use it for any profit-making activity or commercial gain

- You may freely distribute the URL identifying the publication in the public portal

If you believe that this document breaches copyright please contact us providing details, and we will remove access to the work immediately and investigate your claim. 


\title{
Estimating bolt tightness from transverse natural frequencies
}

\author{
Si Mohamed Sah*1, Jon Juel Thomsen ${ }^{1}$, Marie Brøns ${ }^{1}$, Alexander Fidlin², \\ and Dmitri Tcherniak ${ }^{3}$ \\ ${ }^{1}$ Department of Mechanical Engineering, Technical University of Denmark \\ ${ }^{2}$ Department of Engineering Mechanics, Karlsruhe Institute of Technology, \\ Germany \\ ${ }^{3}$ Brüel \& Kjær Sound \& Vibration Measurement, Denmark
}

April 30, 2018

\section{Abstract}

Structural health monitoring techniques based on vibration measurements have been receiving large attention in the last decades, including techniques for estimating bolted joint tightness and detecting loosened bolts. Due to the exposure of bolted joints to external forces, the bolts may loosen and therefore affect healthy functioning of the bolted structure. In this work a technique is proposed to estimate the level of bolt tightness and to quantify the tension based on the measured natural frequencies of the bolt, in particular the first transverse natural frequency. An experiment is performed on two structure specimens each clamped with a bolt of different length. The bolts bending vibrations are excited by impacting the bolts head along the transverse direction. The excited transverse natural frequencies are then recorded as the bolts are gradually tightened. The measured frequencies trends are explained by modeling the bolt as a pre-stressed one dimensional beam with elastic supports at both ends. The experimental results are reproduced using an analytical function that expresses the boundaries stiffness in terms of the bolt tension. The sensitivity of the measured bolt first transverse natural frequency demonstrates the potential of this frequency-based technique in estimating bolt tightness.

\footnotetext{
*smsah@mek.dtu.dk
} 


\section{Introduction}

Bolted joints are widely used in engineering structures and machine design, like wind turbines, drilling rigs, bridges and engines, just to name a few. When a structure with bolted joints is exposed to dynamic loads in the form of shock, vibration or cyclic thermal loading, the fasteners may turn loose [1]. In the case of vibration loading, when the external force overcomes the clamping friction between the bolt and nut, this leads to a relative movement between these two surfaces to occur which results in loosening of the fastener [2]. Such loosening may lead to structural failures if not discovered in time, which can be catastrophic in safety critical applications. In 2015 a 119 meter tall wind turbine located in the Lemnhult wind farm near Vetlanda, Sweden collapsed. The cause was attributed to insufficient clamping forces in the joints, which was overcome by the wind forces. Therefore, a regular checking and documentation of proper bolt tightness is essential for certification and safe operation.

Several methods can be used to estimate bolt tension. These include torque control, angle control, stretch control and the ultrasonic method. Torque control utilizes the torque-tension relation, which is an estimate of how torque provided by the torque wrench is converted into bolt tension [3]. Uncertainties as unknown plastic deformation of the threads, torsion and friction between bolt-head/nut and surface entail a variation in the torque-tension relation of up to $30 \%$ [3]. However due to the simplicity of the torque wrench, this is still the most used method. The angle control method [4] uses the linear relationship between the rotation angle and axial force in an elastic region; however, it is hard to find the elastic region in an actual experiment. Strain gauges are used in stretch control to determine the elongation during bolt tightening and hereby estimate the bolt tension [3]. The accuracy of the strain gauge is high, however due to the requirement of installing these strain gauges, this method may not be practical in bolted assemblies with many fasteners. A discussion on the various bolt-tightening verification techniques can be found in [5].

The ultrasonic method utilizes the relationship between acoustic wave velocity and length of the bolt combined with the stress state in bolt [6-8]. A transducer emits an ultrasound impulse from one end of the bolt, and the travel time back and forth of the impulse is then recorded to estimate the tension in the bolt [9]. Most ultrasonic devices in industry use the longitudinal waves to determine bolt tension (mono-wave method); however researchers have been working in using also transverse waves (bi-wave method): A velocity ratio approach, which uses a velocity ratio between the longitudinal and the transverse waves that have different acoustoelastic coefficients, was suggested to estimate the tightening force of a bolt [10]. With this method, the time of flight measurement in the unstressed state is not needed. Ultrasonic techniques have also been used to study contact pressure distribution at the clamped interface of bolted joints $[11,12]$. It was further shown that ultrasonic reflections from the clamped interface could be used to estimate the progress of relaxation in bolted joints [13]. The ultrasonic method provides a better accuracy compared to the torque wrench, and also determines bolt tension without any impact on the joint stiffness, as opposed to strain gauges. However, besides stress, the method is also influenced by temperature and by plastic elongation of the bolt [14]. There are also some requirements for the method to be 
used, e.g. the bolt ends must be flat, parallel and have a reasonable surface finish, and bolts with short effective lengths are not suitable.

In recent years, a continuous interest was placed on improving and developing other techniques to assess the loosening/tightening health state of bolted joints. Among these techniques are those based on vibration measurements, where the structural vibration response is analyzed for detecting failure in bolted joints. Todd et al [15] investigated the effectiveness of monitoring changes in structural frequencies and mode shapes, and found that these properties may not be ideal to track joint functionality loss. Meyer and Adams [16] investigated an impact modulation testing method to identify loose bolts within a structure. They developed a modulation index based on the amplitudes of the side-bands in the response spectrum, and showed that this index increased as the bolt torque decreased. Other methods also exploited the nonlinear dynamics of bolted joints: Amerini et al [17] investigated a vibro-acoustic modulation method, where two indices were developed to assess the health state of bolted joint; A hyperbolic tangent function was proposed to approximate the experimental data with excellent correlation. Milanese et a. [18] developed a method that uses output-only vibration data. They showed that the bolted joint exhibits linear behavior when it is tight, while nonlinearly when loose. This nonlinearity was attributed to nonlinear boundaries stiffness of the composite beam. Paez et al. [19] experimentally found the natural frequency of a bolted joint to exhibits a nonlinear dependency on the boundary stiffness, and tending to an asymptotic value for larger stiffness.

With the above-mentioned methods, the assessment of the bolted joint tightness is based on the vibration response of the structure. In this work, by contrast a technique based on the vibration response of the bolt itself, specifically its transverse natural frequency, is presented. This frequency-based technique has two objectives: assess the bolt tightness level (i.e. loose or tight), and quantify the bolt tension. Experimental and theoretical studies are performed to test and support the reliability of this technique. Two bolts of different lengths are considered in the experiment, where each bolt is used to clamp a test structure. The bending vibration of the bolt is excited by impacting the bolt-head along the transverse direction. The transverse natural frequencies of the bolt are then measured as the bolt is gradually tightened up to $70 \%$ of its yield force, i.e. to about nominal tightness. The measured frequencies trends for the two bolts are then theoretically investigated by considering a simple model that consists of a pre-stressed one dimensional beam with elastic supports at both ends. The boundaries stiffness of the beam are expressed analytically as function of the bolt tension. This theoretical model helps understand the measured frequencies trends and shows that the bolt tension can be quantified from the measured frequency. Section 2 introduces the experimental set up and presents the measured data. Section 3 investigates theoretically the obtained experimental data. Section 4 combines the results of the two previous sections and discusses them, and Section 5 presents conclusions. 


\section{Experimental observations}

\subsection{Test rigs and signal instrumentation}

The two test specimens $\varnothing 100 \times 100 \mathrm{~mm}$ (semi-cylindrical) and $\varnothing 100 \times 200 \mathrm{~mm}$ (cylindrical) (Figure 1a,b) were manufactured from alimunium (Young's modulus 70 GPa, density 2700 $\mathrm{kg} / \mathrm{m}^{3}$ ) with a bore for a bolt in each one. Two steel bolts (Young's modulus $207 \mathrm{GPa}$, density $7850 \mathrm{~kg} / \mathrm{m}^{3}$ ) of grade 8.8 were used (Figure 1c): M12×260 mm for the long test rig and $\mathrm{M} 12 \times 140 \mathrm{~mm}$ for the short one. The diameter of the clearance holes in the test rigs was $14 \mathrm{~mm}$ to avoid contact between the test rigs and mounted bolts. The clearance considered here corresponds to a fit between normal and loose, [20]. Each test rig was designed such that its first transverse natural frequency was larger than the two first transverse natural frequencies of their respective bolt. The combined test rig and bolt system was put on a foam to approximate free-free boundary conditions.

A force sensor washer (Type KMR $\varnothing 12 \mathrm{~mm}$ from HBM), placed underneath the bolt head, was used to measure the axial force between bolt-head and the structure flange. Two hardened washers were placed at both sides of the force washer to help distribute the clamping force all over its surface (Figure $2 \mathrm{~b}$ ). The force washer was connected to an amplifier (Type AE301S7 from HBM) and the amplifier to a voltmeter to read off the voltage. The force washer was calibrated using a tension test machine (Instron 8502 testing system) for $5 \mathrm{~V}$ output to equal $60 \mathrm{kN}$ of load. The relationship between applied load and the output voltage was found to be linear in the range of relevance, which was up to $70 \%$ of the bolt yield force [3]. In the rest of the paper, the clamping force between bolt-head/nut and structure flanges, and the bolt tension have the same meaning, while load refers to the pre-load applied by the tightening device.

An impact pendulum was designed (Figures 1d and 2a) to impact the bolt-head along the transverse direction of the bolt in a controllable manner through a steel tip. A force transducer (B\&K Type 8206) was mounted between the tip and the pendulum arm to measure the applied impacting force. On the bolt-head an accelerometer (B\&K Type 4397) was glued in the transverse direction using a thin layer of beeswax (Figure 2b). The accelerometer and the force transducer were connected to B\&K DAQ LAN-Xi module Type 3150.

\section{$2.2 \quad$ Test procedure}

The two bolts, the long M12×260 $\mathrm{mm}$ and the short M12×140 mm, were gradually tightened up to $70 \%$ of their yield force, around $40 \mathrm{kN}$, using a hydraulic tensioner (SKF) [21]. The hydraulic tensioner was chosen over the torque wrench to avoid deforming the threads and to minimize the friction between the nut and the bolt threads. This way the same geometric and material properties of the bolt were retained through the continuous loading-unloading cycles, and therefore the variability, usually caused by the torque wrench, was eliminated. During the tightening process the tensioner was hand pump pressurized, and while the bolt was elongated, the nut was finger tightened. The pressure was then released and hereby the test rig flanges compressed against the bolt-head/nut surfaces [22]. Once certain tension level 

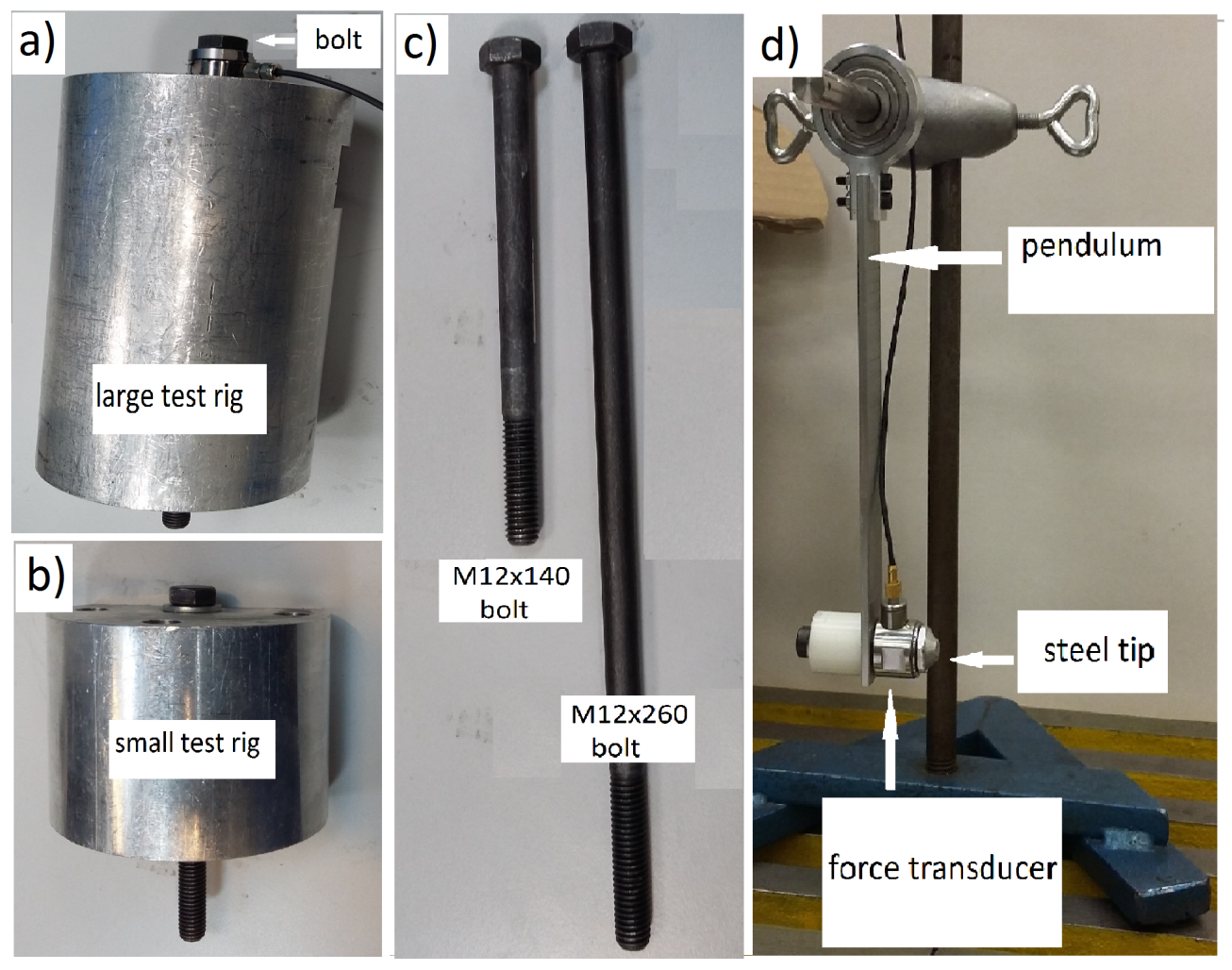

Figure 1: a,b) Test specimens. c) Bolts. d) Impact pendulum.

was reached, an impact modal testing was performed. Then the bolt was loosened using the hydraulic tension and again tightened with a higher tension level for another impact testing.

The bolt was excited along the transverse direction by means of the impact pendulum. The resulting transverse vibrations response was sensed using the accelerometer mounted on the bolt-head. The excitation and response signals were recorded simultaneously using B\&K PULSE Labshop software, and then processed with a frequency span and resolution of 13.1 $\mathrm{kHz}$ and $1 \mathrm{~Hz}$, respectively. At each level of tension the bolt was impacted three times and the results were averaged to obtain one frequency response function (FRF). The transverse natural frequencies were determined by localizing peaks in the FRF. These frequencies can be considered as accurate measures of the undamped ones, since the damping is so low $(<1 \%)$. This procedure was repeated for each tension level until reaching $70 \%$ of the bolt yield force, and the data collected was referred to as a Test. Two Tests were performed, and in each one, the tension level was increased until reaching approximately $40 \mathrm{kN}$. The first two tension levels in each Test were reached using hand tightening to obtain very low values of tension levels.

Figure 3 shows an example of FRF and coherence plot for a bolt tension $(26 \mathrm{kN})$, where the first two peaks correspond to the bolts first two natural transverse frequencies. The good coherence, close to unity, in the frequency range including the first two natural frequencies 

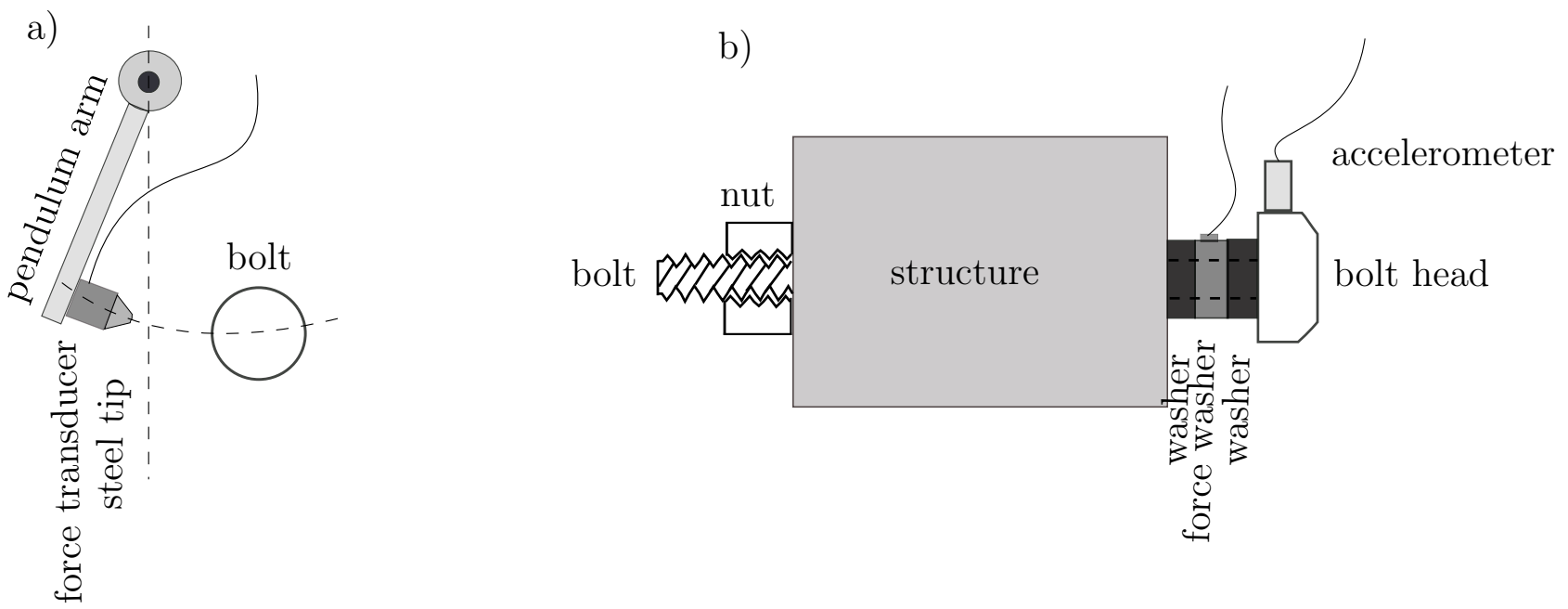

Figure 2: Schematic impact pendulum (a) and bolted structure (b).
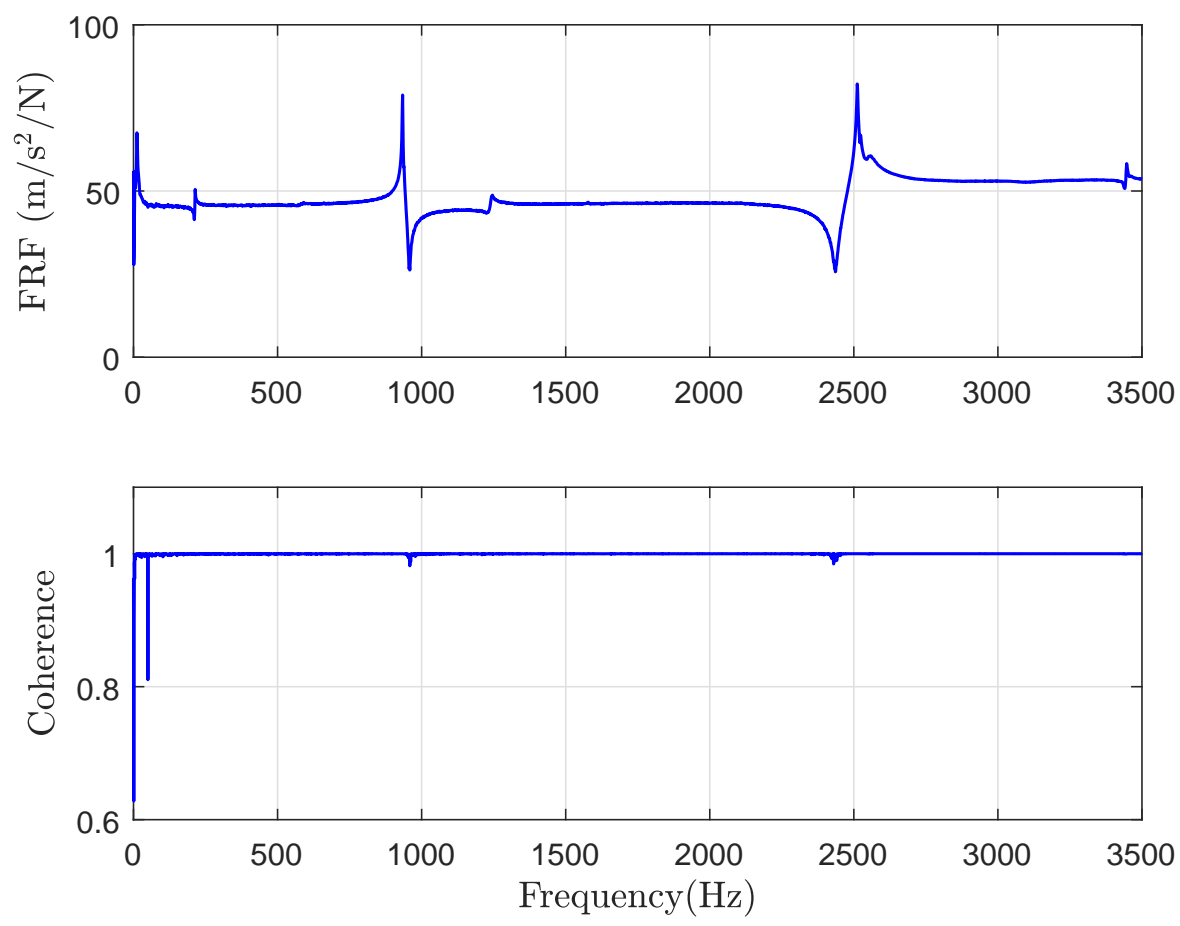

Figure 3: Measured FRF (top) and coherence (bottom) with transverse impact for the M12 $\times 260 \mathrm{~mm}$ bolt at tension $(26 \mathrm{kN})$.

indicates the response is mainly due to the impact, and that the relationship between input and output is linear with a good signal to noise ratio. 


\subsection{Test results}

Figure 4 shows the squared transverse natural frequencies for the two bolts as a function of bolt tension. The measured frequencies for the first two modes, $f_{1}$ and $f_{2}$, are normalized by the respective theoretical frequencies for a corresponding clamped-clamped uniform beam, $f_{1, c c}$ and $f_{2, c c}$, with $f_{i, c c}=1 /(2 \pi) \sqrt{\lambda_{i, c c}^{4} E I /\left(l^{4} \rho A\right)}$, and where $E I$ is the bending stiffness, $\rho A$ the mass per unit length and $l$ the corresponding effective length for each bolt, and $\left(\lambda_{1, c c} \approx 4.73, \lambda_{2, c c} \approx 7.85\right)$. The tension, which corresponds to the clamping force between the test rig flanges and the bolt-head/nut surfaces, is normalized by the transverse stiffness $E I / l^{2}$ for each bolt. In Appendix, we provide the symbols and notations used in this work.

In the case of the long bolt, as shown in Figure 4a, at low tension the squared first transverse natural frequency changes nonlinearly with tension. As the bolt is gradually tightened the squared frequency starts changing linearly with tension. This linear behavior starts approximately when the ratio $f_{1} / f_{1, c c}=1$ indicating that the pre-stressed bolt starts behaving as if it has clamped-clamped boundary conditions. The nonlinear-to-linear change was also observed by other authors who analyzed the vibration response of a structure, e.g. [18,23]; the nonlinear behavior was attributed to the nonlinear boundaries stiffness [18].

For the second vibration mode, the squared frequency shows the same trend as for the first mode, see Figure 4c. However, the change in the squared frequency is less sensitive to the tension. This suggests that the first transverse mode is a better indicator of the bolt tightness than the second. Therefore the frequency-based method presented in this work relies on the measured first transverse natural frequency of the bolt, and the forthcoming analytical investigation aims primarily to recover and explain the experimental frequency trends of the first mode.

For the short bolt, the same remarks can be noted as in the case of the long bolt, see Figure 4b,d. However the transverse natural frequency of the short bolt is less sensitive to tension than the long bolt. Also the tension $p_{c c}$ at which the ratio $f_{1} / f_{1, c c}=1$, i.e. where the bolt starts behaving as a pre-stressed clamped-clamped beam, differs between the long and short bolts. The bolt tension value corresponding to $p_{c c}$ is lower for the long bolt $(\approx 10$ $\mathrm{kN})$ compared to the one for the short bolt $(\approx 20 \mathrm{kN})$, indicating that the long bolt becomes effectively clamped at lower tension than the short bolt. An explanation of this observation is provided in the theoretical analysis.

The nonlinearity, in the squared natural frequency versus tension relation, observed for both bolts is attributed to the boundaries, specifically in the contact between the structure flanges and the bolt-head/nut surfaces. When the clamping force at the interfaces is low and the bolt is struck, both the bolt-head and the nut move against the structure which induce friction, however the effect of the boundary damping on the frequency is minimal. Therefore the dominant effect of the boundaries on the transverse frequency comes from the stiffness. In the next section, the effect of the boundaries stiffness on the frequency is theoretically investigated for low and high tension. 

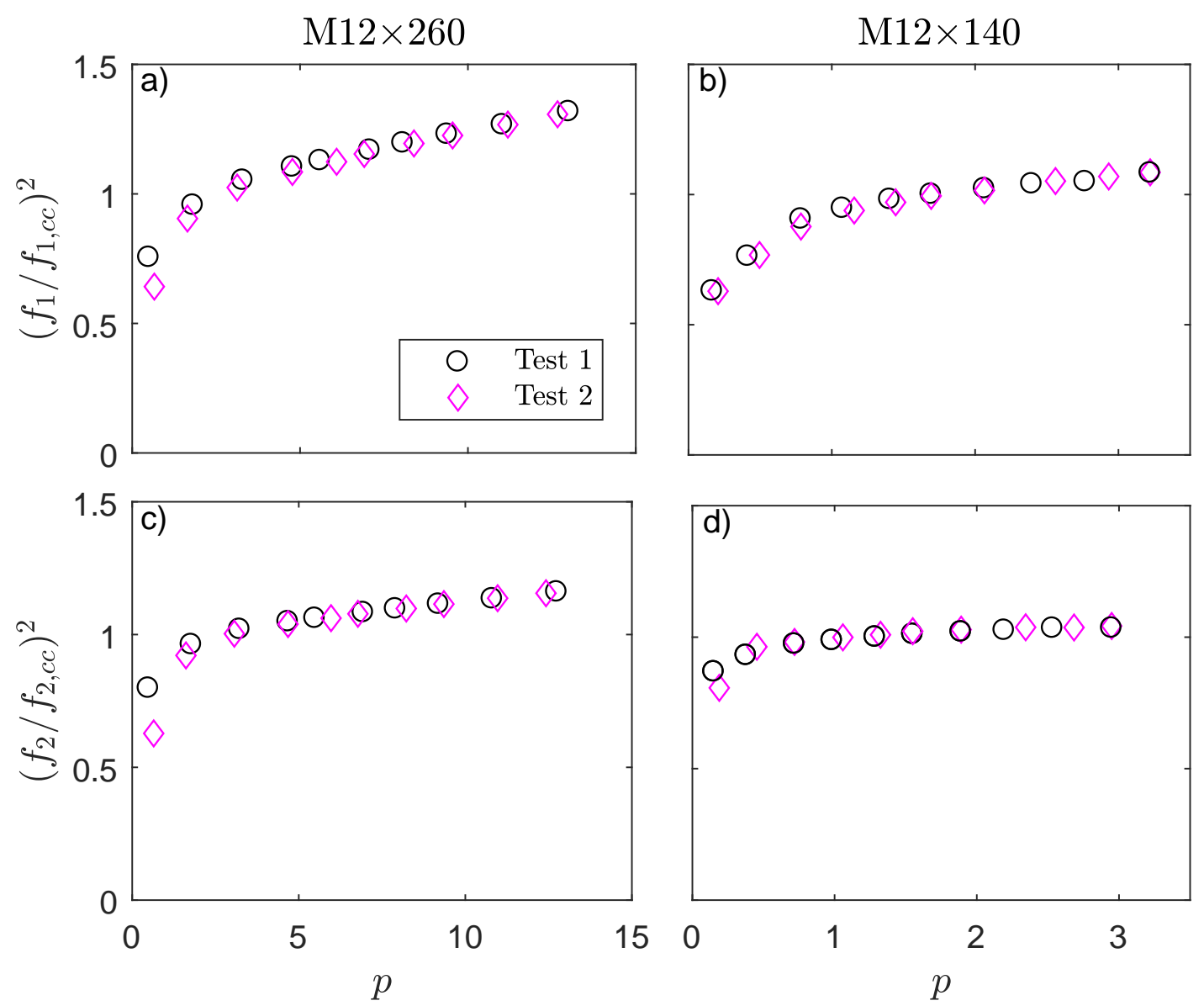

Figure 4: Measured first and second nondimensional squared transverse natural frequency of the long bolt M12×260 (a,c) and short bolt M12×140 (b,d) as function of nondimenisonal tension $p$. (a,b) first mode, $(\mathrm{c}, \mathrm{d})$ second mode.

\section{Theory}

Here we suggest a theory to help explain the behavior of the bolt frequencies trends for low as well as high tensions (Figure 4). It was shown in the previous section that at high tension the bolt behaves as if clamped at both ends; this suggests the bolt can be modeled as an axially pre-stressed clamped-clamped beam, i.e. where the boundary stiffness is infinite. For low tension, a model of one-dimensional beam with finite values of boundary stiffness is considered to explain the experimental data. The predictions of this model should approach the data at high tension, namely pre-stressed clamped-clamped beam, when the boundaries stiffness become infinite. Hereby the theoretical model "full model" can cover all tension levels, and explain data from low to high tensions. The tightened bolt is modeled as a beam having translational $\tilde{k}_{u}$ and rotational $\tilde{k}_{\theta}$ stiffness at both ends, and subjected to a tensile 


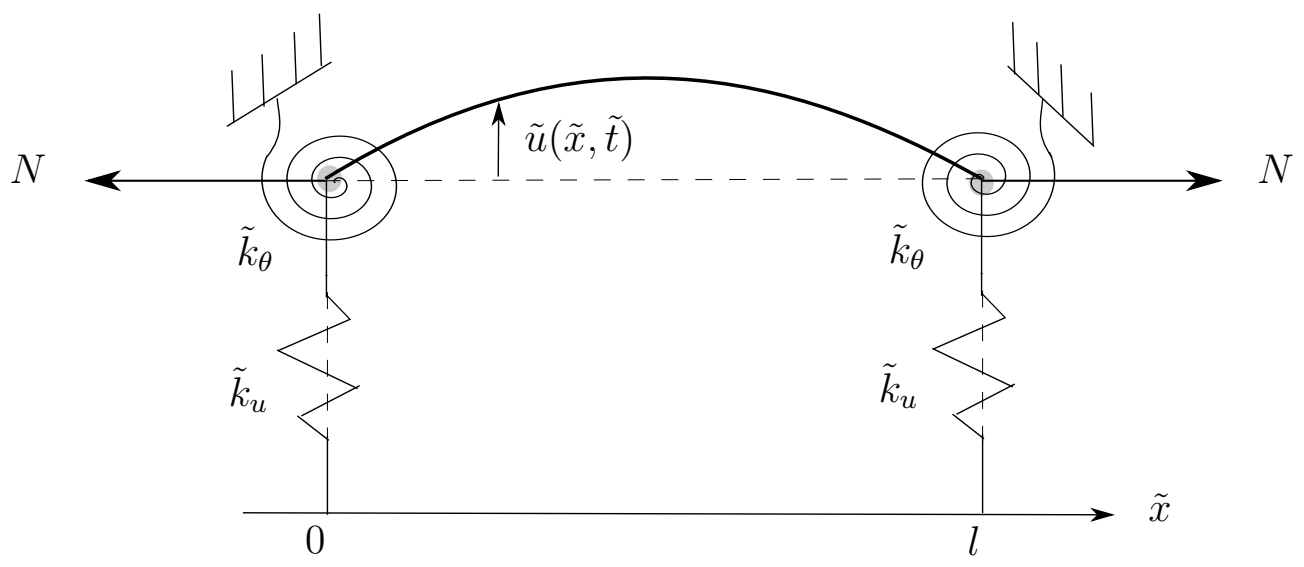

Figure 5: Simple bolt model - a tensioned slender beam with finite boundary stiffness.

axial force $N$, see Figure 5. Although for bolts the bending stiffness has the main effect on the transverse vibrations of the beam, the boundary stiffness as characterized by the three parameters $\tilde{k}_{u}, \tilde{k}_{\theta}$ and $N$ are expected to also have some effect. The equation of motion of the beam transverse deflection $\tilde{u}(\tilde{x}, \tilde{t})$ is obtained using Bernoulli-Euler theory, where $\tilde{x}$ and $\tilde{t}$ denote the longitudinal coordinate and the time, respectively.

\subsection{Equation of motion}

The Bernoulli-Euler equation for the pre-stressed beam has the nondimensional form [24,25]:

$$
\ddot{u}+u^{\prime \prime \prime \prime}-p u^{\prime \prime}=0
$$

where $u=u(x, t)$ is the transverse deflection at time $t$ and $x \in[0,1]$ is the axial coordinate. The boundary conditions are:

$$
\begin{aligned}
& -u^{\prime \prime \prime}(0, t)+p u^{\prime}(0, t)=k_{u} u(0, t), \\
& u^{\prime \prime}(0, t)=k_{\theta} u^{\prime}(0, t), \\
& -u^{\prime \prime \prime}(1, t)+p u^{\prime}(1, t)=-k_{u} u(1, t), \\
& u^{\prime \prime}(1, t)=-k_{\theta} u^{\prime}(1, t),
\end{aligned}
$$

and all parameters and variables are nondimensional:

$$
\begin{aligned}
& x=\frac{\tilde{x}}{l}, \quad t=w_{0} \tilde{t}, \quad u=\frac{\tilde{u}}{l}, \\
& p=\frac{N l^{2}}{E I}, \quad k_{u}=\frac{\tilde{k}_{u} l^{3}}{E I}, \quad k_{\theta}=\frac{\tilde{k}_{\theta} l}{E I} .
\end{aligned}
$$

In Eqs. (1)-(5) dots and primes denote differentiation w.r.t. to $t$ and $x$, respectively, subscripts $u$ and $\theta$ denote "translational" and "rotational", respectively. The axial coordinate $x$ and 
transverse deflection $u$ are nondimensionalized by the beam length $l$, which models the effective length of the bolt. Time $t$ is nondimensionalized by the characteristic frequency $w_{0}=\sqrt{E I / \rho A l^{4}}$. A solution form $u(x, t)=\phi(x) \cos (w t)$ inserted in Eqs. (1)-(5) gives

$$
\begin{aligned}
& \phi^{\prime \prime \prime \prime}(x)-p \phi^{\prime \prime}(x)-w^{2} \phi(x)=0, \\
& -\phi^{\prime \prime \prime}(0)+p \phi^{\prime}(0, t)=k_{u} \phi(0), \\
& \phi^{\prime \prime}(0)=k_{\theta} \phi^{\prime}(0), \\
& -\phi^{\prime \prime \prime}(1)+p \phi^{\prime}(1)=-k_{u} \phi(1), \\
& \phi^{\prime \prime}(1)=-k_{\theta} \phi^{\prime}(1) .
\end{aligned}
$$

The general solution of Eq. (7) can be written:

$$
\phi(x)=C_{1} \cos \lambda x+C_{2} \sin \lambda x+C_{3} \cosh \gamma x+C_{4} \sinh \gamma x,
$$

where $\lambda$ and $\gamma$ are given by

$$
\lambda=\sqrt{\sqrt{\left(\frac{p}{2}\right)^{2}+w^{2}}-\frac{p}{2}} \quad \text { and } \quad \gamma=\sqrt{\sqrt{\left(\frac{p}{2}\right)^{2}+w^{2}}+\frac{p}{2}},
$$

satisfying

$$
\gamma=\sqrt{\lambda^{2}+p}
$$

and where $C_{1}, C_{2}, C_{3}$ and $C_{4}$ are constants to be determined from the boundary conditions Eqs. (8)-(11). Inserting Eq. (12) into Eqs.(8)-(11) and solving for non-zero $\mathbf{C}=$ $\left\{\begin{array}{llll}C_{1} & C_{2} & C_{3} & C_{4}\end{array}\right\}^{T}$, by requiring the determinant to vanish, the frequency equation is obtained:

$$
\begin{aligned}
& {\left[\left(4 w^{2}+p^{2}\right)\left(w^{2} k_{\theta}^{2}+k_{u}^{2}\right)+p w^{2}\left(3 w^{2}+p^{2}\right)-2 k_{u} k_{\theta} p w^{2}-k_{u}^{2} k_{\theta}^{2} p\right] \sin \lambda \sinh \gamma} \\
& +\left[\left(w^{2}-k_{u} k_{\theta}\right)^{2}-2 k_{u} k_{\theta}\left(2 w^{2}+p^{2}\right)\right] \cos \lambda \cosh \gamma \\
& \frac{1}{\sqrt{2}} \sqrt{\sqrt{4 w^{2}+p^{2}}+p}\left(\sqrt{4 w^{2}+p^{2}}\left[k_{\theta} p w^{2}+k_{u}\left(2 w^{2}+p\right)+k_{u} k_{\theta}\left(k_{u} p-2 k_{u}\right)\right]\right. \\
& \left.+\left(4 w^{2}+p^{2}\right)\left(k_{\theta} w^{2}-k_{u} p-k_{\theta}^{2} k_{u}\right)\right) \sin \lambda \cosh \gamma \\
& -\frac{1}{\sqrt{2}} \sqrt{\sqrt{4 w^{2}+p^{2}}+p}\left(\sqrt{4 w^{2}+p^{2}}\left[k_{\theta} p w^{2}+k_{u}\left(2 w^{2}+p\right)+k_{u} k_{\theta}\left(k_{u} p-2 k_{u}\right)\right]\right. \\
& \left.-\left(4 w^{2}+p^{2}\right)\left(k_{\theta} w^{2}-k_{u} p-k_{\theta}^{2} k_{u}\right)\right) \cos \lambda \sinh \gamma-2 w\left(w^{2}+k_{u} k_{\theta}\right)=0 .
\end{aligned}
$$

The frequency equation can be reduced to that of a pre-stressed clamped-clamped beam [26] by dividing Eq. (15) by $k_{\theta}^{2} k_{u}^{2}$ and letting $k_{\theta}$ and $k_{u}$ approach infinity, which gives:

$$
2 w \cos \lambda \cosh \gamma-p \sin \lambda \sinh \gamma-2 w=0,
$$

where $\lambda$, in this case, slightly changes with tension $p$, resulting in a linear dependency of the squared frequency on tension. The expression of the frequency is given by combining Eqs. (13) and (14) such that:

$$
w^{2}=\lambda^{4}\left(1+p / \lambda^{2}\right)
$$


The frequency $w$ is obtained by solving Eq. (15), which is function of the tension $p$, and the rotational and translational stiffness, $k_{\theta}$ and $k_{u}$ respectively. In this study, the tension $p$ is provided by the force washer underneath the bolt-head, while the boundary stiffness is theoretically investigated in the following.

\subsection{Boundary stiffness vs. tension}

In this section analytical expressions for the rotational $k_{\theta}$ and translational $k_{u}$ stiffness are investigated. The boundary stiffness expressions are sought on the fact that real engineering surfaces are to some extent rough. This roughness is manifested as a distribution of tiny asperities at a micro-scale level. Figure 6 shows a schematic of the bolt-head/nut and the structure surfaces clamped together. The asperity interactions of the interface can be thought of as springs connecting the mating surfaces, as shown in Figure 6b. Drinkwater et al. [27] observed, using a spring model, that the normal (interfacial) stiffness increases as the contact pressure is increased. No exact relationship exists between normal stiffness and contact pressure; however Greenwood and Williamson [28] introduced a statistical model for the contact between a rough and plane surfaces. They derived an expression for the contact force assuming the asperities summit to be spherical with constant radius and their heights following a Gaussian distribution:

$$
N=\frac{4}{3} E^{*} \eta \beta^{1 / 2} \sigma^{3 / 2} \int_{h}^{\infty}(s-h)^{3 / 2} \phi(s) d s,
$$

where $\eta$ is the asperity density, $\beta$ the asperity radius of curvature, $E^{*}$ the composite modulus of elasticity of the material of the two surfaces, $\sigma$ the composite standard deviation of asperity height distributions and $h=d / \sigma$ the normalized gap $d$ between the two surfaces. The height distribution $\phi(s)=\exp \left(-s^{2} / 2\right)$ is scaled so that its standard deviation is unity. The contact (clamping) force $N$ in Eq. (18) corresponds to the tensile force $N$ in the beam (cf. Figure 5), which models the bolt tension. The normal stiffness can be deduced from Eq. (18), since:

$$
\tilde{k}_{n}=\left|\frac{\mathrm{d} N}{\mathrm{~d} \delta_{n}}\right|=\frac{1}{\sigma} \frac{\mathrm{d} N}{\mathrm{~d} h}=2 E^{*} \eta \beta^{1 / 2} \sigma^{1 / 2} \int_{h}^{\infty}(s-h)^{1 / 2} \phi(s) \mathrm{d} s,
$$

where $\delta_{n}$ denotes the deformation induced by the contact force $N$. Eq. (19) shows that the stiffness is dependent on the surface topography through the parameters $\eta, \beta$ and $\sigma$. It was theoretically shown [29] that these parameters have an important effect on the normal stiffness. Figure 7 shows the normal stiffness $\tilde{k}_{n}$ as function of the contact force $N$ obtained by Eqs. (18) and (19), using numerical integration.

The bending vibration of the bolt causes an uneven contact force distribution along the contacting surfaces between the bolt-head/nut and structure flanges, which results in these surfaces being titled with respect to each other, Figure 8a. Therefore the normal stiffness induces also a rotational stiffness at the bolt ends (bolt-head and nut). Assuming that the normal stiffness is homogeneously distributed over the contact ring in Figure 8b, which models the bolt-head/nut surfaces, the normal force $\mathrm{d} N$ on an area infinitesimal $\mathrm{d} a$ is: 
a)

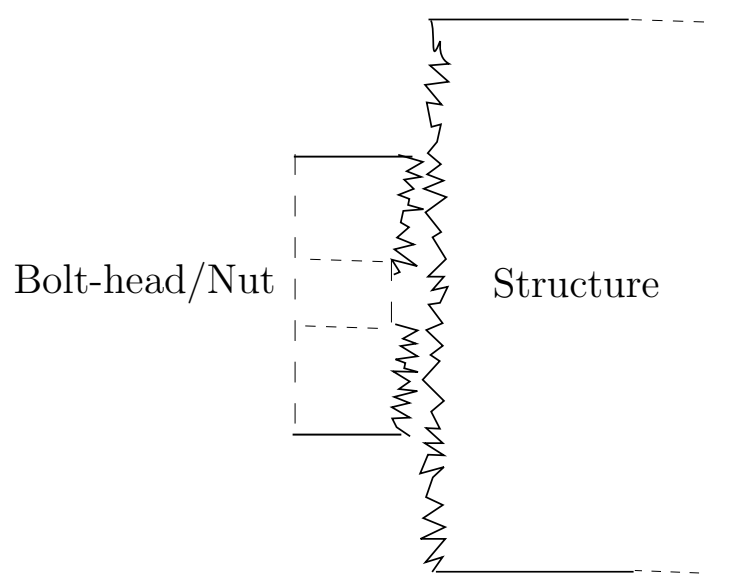

b)

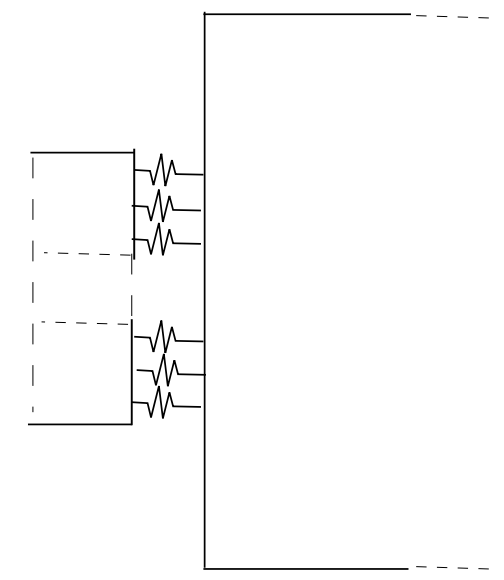

Figure 6: Contact between structure flange and bolt-head/nut surface.

$$
\mathrm{d} N=\tilde{K}_{n} \delta_{\theta} \mathrm{d} a=\frac{\tilde{k}_{n}}{\pi\left(R^{2}-r^{2}\right)} \rho \sin \phi \sin \theta \mathrm{d} a,
$$

where $(\rho, \phi)$ are the polar coordinates of $\mathrm{d} a$ in the ring surface, and $r$ and $R$ the inner and outer radius of the bolt-head/nut, respectively, (cf. Figure 8b). The term $\delta_{\theta}=\rho \sin \phi \sin \alpha$ denotes the deformation induced by tilting of the ring, and $\tilde{K}_{n}=\tilde{k}_{n} / \pi\left(R^{2}-r^{2}\right)$ the normal stiffness density. The torque about the axis of abscissas in Figure $8 \mathrm{~b}$ is:

$$
\mathrm{d} M=\mathrm{d} N \rho \sin \phi=\frac{\tilde{k}_{n}}{\pi\left(R^{2}-r^{2}\right)} \rho^{2} \sin ^{2} \phi \sin \theta \mathrm{d} a .
$$

Integrating over the surface of the ring, we obtain the total torque $M$ about the axis of abscissas:

$$
\begin{aligned}
M & =\int_{r}^{R} \int_{0}^{2 \pi} \mathrm{d} M=\int_{r}^{R} \int_{0}^{2 \pi} \frac{\tilde{k}_{n}}{\pi\left(R^{2}-r^{2}\right)} \rho^{2} \sin ^{2} \phi \sin \theta \rho \mathrm{d} \rho \mathrm{d} \phi \\
& =\frac{\sin \theta}{4}\left(R^{2}+r^{2}\right) \tilde{k}_{n},
\end{aligned}
$$

and differentiating with respect to $\theta$ gives the rotational stiffness:

$$
\tilde{k}_{\theta}=\frac{\mathrm{d} M}{\mathrm{~d} \theta}=\frac{\cos \theta}{4}\left(R^{2}+r^{2}\right) \tilde{k}_{n}
$$

The translational stiffness on the other hand can be calculated as [30]: 


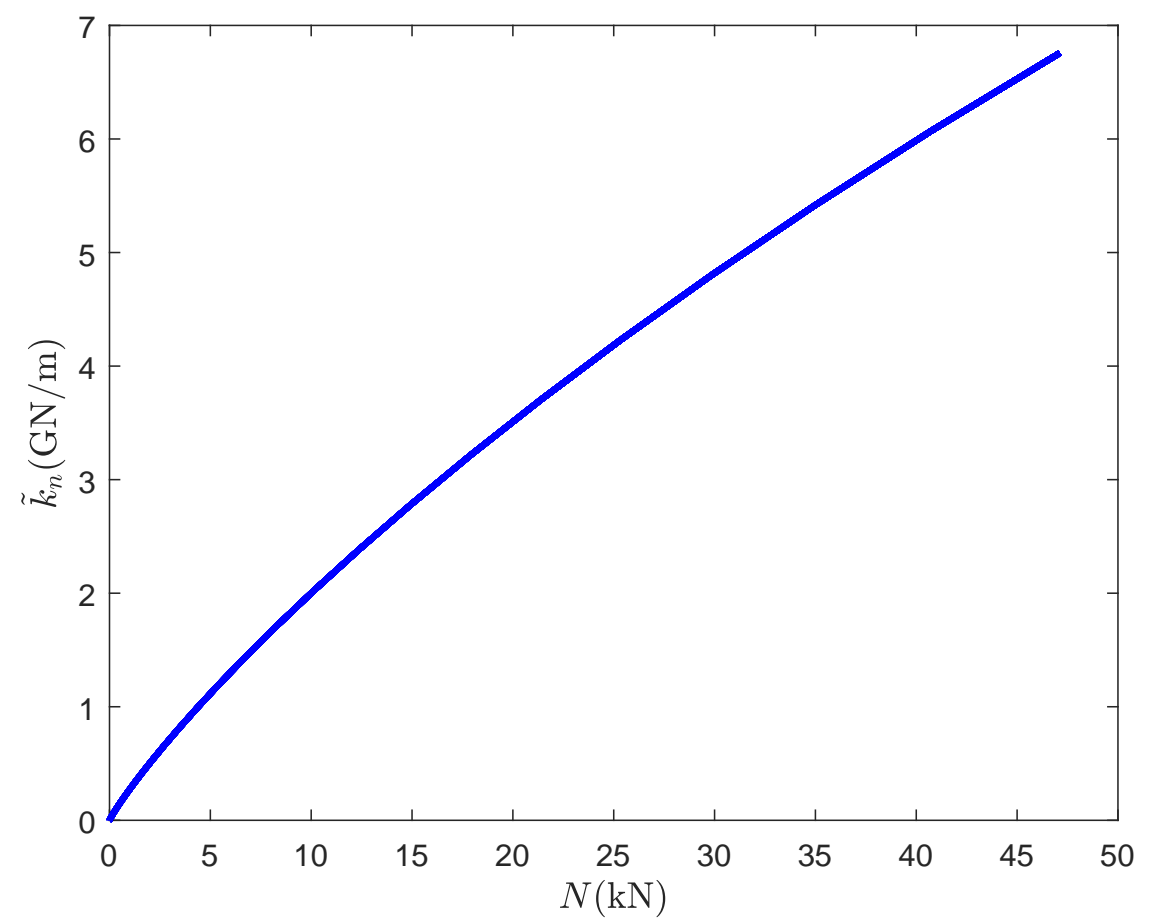

Figure 7: Normal stiffness, Eq. (19), vs. clamping force, Eq. (18) with physical parameters taken from $[29]$.

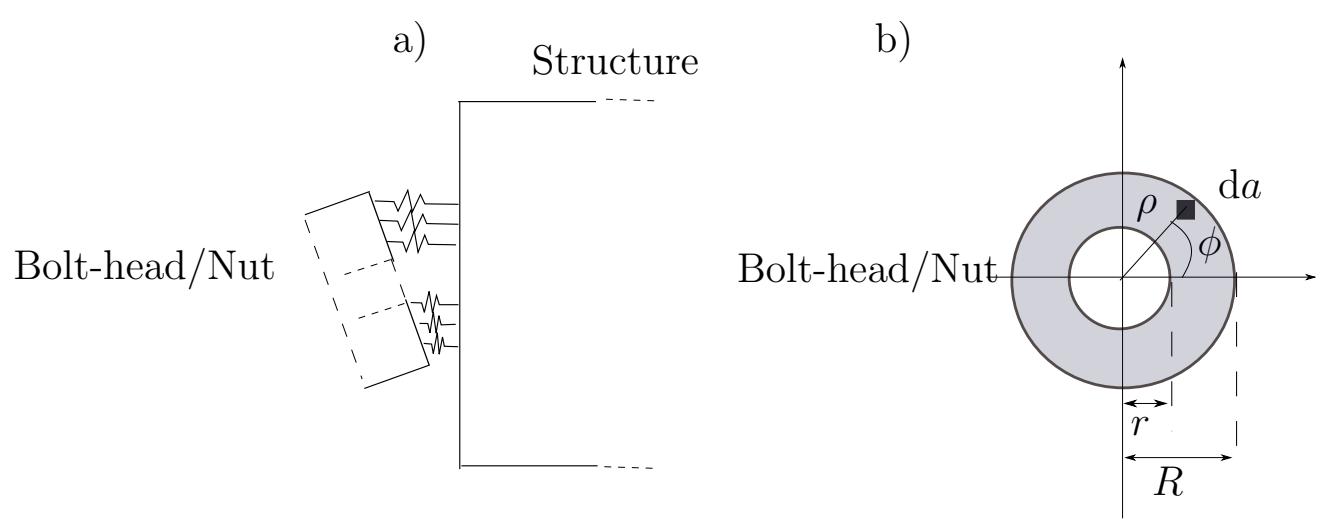

Figure 8: a) Tilted mating surfaces. b) Bolt-head/Nut surface geometry. 


$$
\tilde{k}_{u}=\frac{C(1-\nu)}{(2-\nu)} \tilde{k}_{n}
$$

where $\nu$ is the Poisson ratio and $C=\pi / 2$. Eq. (25) was obtained by relating the elastic tangential displacement of an asperity to a tangential force, and to the radius of the contact area of an asperity, and then integrating for all asperities assuming a Gaussian distribution. The tangential stiffness can result from the contact of an asperity shoulder with an another asperity shoulder on the opposite surface. Other expressions similar to Eq. (25) were derived by other authors $[31,32]$ with the only difference the value of the factor $C$.

Eqs. (24) and (25) show that the rotational and the translational stiffness are both proportional to the normal stiffness $\tilde{k}_{n}$ with factors that depend on the geometry and the material properties of the mating surfaces. In what follows, the data represented in Figure 4 are analytically investigated using the expressions of $\tilde{k}_{\theta}$ and $\tilde{k}_{u}$ given in Eqs. (24) and (25), together with the nondimensionlized tension $p$ which is related to $N$ (cf. Eq. (6)).

\subsection{Natural frequency vs. tension}

The effect of tension and boundary stiffness on the transverse natural frequencies of the beam can be investigated by first rewriting Eq. (15) such that it is function of $\lambda, k_{\theta}, k_{u}$ and $p$, using Eqs. (14) and (17):

$$
g\left(\lambda, p, k_{\theta}, k_{u}\right)=0
$$

solving numerically for the roots $\lambda$, and then using Eq. (17) to obtain the squared natural frequency $w^{2}$. Furthermore, Eqs. (18),(19),(24), and (25) provide an implicit relation between $k_{\theta}, k_{u}$ and $p$; however a closed form expression relating the stiffenss to the tension is desired to better perceive and understand the effect of boundary stiffness on natural frequencies. Based on the analytical expressions, Eqs. (18) and (19), Figure 7, and experimental observations (e.g. $[27,33]$ ), an approximating function for the normal stiffness $k_{n}$ is proposed:

$$
k_{n}=\frac{c_{1} \tanh \left(c_{2} p\right)}{1+c_{3} e^{-c_{4} p}}
$$

where $c_{1}, c_{2}, c_{3}$ and $c_{4}$ are positive constants with no physical meaning. The function $\tanh \left(c_{2} p\right)$ tracks the nonlinear behavior of the stiffness as the tension $p$ is changing at low tension values, and also ensures the condition of zero stiffness at zero tension, while the constant $c_{1}$ controls the behavior at the limit for high tension. The term $c_{3} \exp \left(-c_{4} p\right)$ controls the transition of the stiffness behavior between low and high tension. The rotational and translational stiffness in Eqs. (24) and (25) then become:

$$
k_{\theta}=c_{\theta} k_{n} \quad \text { and } \quad k_{u}=c_{u} k_{n},
$$

where 


$$
c_{\theta}=\frac{\cos \theta}{4} \frac{\left(R^{2}+r^{2}\right)}{l^{2}}, \quad c_{u}=\frac{C(1-\nu)}{(2-\nu)} .
$$

Eq. (26) then becomes:

$$
g\left(\lambda, p, c_{\theta}, c_{u}, c_{1}, c_{2}, c_{3}, c_{4}\right)=0
$$

where the parameters $c_{1}, c_{2}, c_{3}$ and $c_{4}$ are to be obtained by fitting the squared frequency to the experimental data. The $\lambda$ 's are obtained by numerically solving Eq. (30) for a set of values of $\left(c_{1}, c_{2}, c_{3}, c_{4}\right)$, and the corresponding frequency solutions, $w=w(p)$, are deduced using Eq. (17), and then compared to the measured frequencies $w_{j}=2 \pi f_{j}$ for every value of the measured bolt tension $p_{j}$, where the subscript $j$ denotes a specific tension level. The parameters $\left(c_{1}, c_{2}, c_{3}, c_{4}\right)$ are obtained so as to minimize the difference between the solution $w(p)$ and the measured frequencies $w_{j}$. The parameter $c_{1}$ controls the limit at high tension, $c_{2}$ the behavior at low tension, and $c_{3}$ and $c_{4}$ control the transition from low to high tension.

The fitted parameters are used to produce Figure 9, which shows the roots $\lambda$ for the first two modes of the frequency equation (30) (blue solid line) in the case of the long bolt M12x260. Figure 9 also shows the roots for the first two modes of the pre-stressed clampedclamped beam $\lambda_{p c c}$ (red dashed line), Eq. (16), which for zero tension $(p=0)$ reduce to the clamped-clamped beam case $\left(\lambda_{c c} \approx 4.73,7.85\right)$. For zero tension, the $\lambda$ 's of Eq. (30) emanate from the zero solution $(\lambda=0)$ of the equation $\cos \lambda \cosh \lambda=1$, which corresponds to rigid body motion of the beam. This is caused by the link between tension and boundary stiffness, which implies zero boundary stiffness for zero tension, with corresponding unrestricted rigid body modes. As the tension $p$ increases, the roots start changing nonlinearly with $p$; however as the value of the tension further increases, the roots start changing close to linearly, in particular the $\lambda$ 's approach the ones for the pre-stressed clamped-clamped beam $\lambda_{p c c}$.

Figure 10 shows the rotational and translational stiffness $k_{\theta}$ and $k_{u}$ calculated using Eq. (28) with fitting parameters $\left(c_{1}, c_{2}, c_{3}, c_{4}\right)$ obtained for the first transverse mode of M12x260 bolt. The rotational and translational stiffness increase as the tension increases. The frequency solution (blue solid line) is produced and plotted against the measured frequencies (markers), as shown in Figure 11, for the first two transverse modes of the two bolts M12x260 and M12x140.

The frequency solution with fitted parameters (blue solid line) for the first mode agrees excellently with the measured frequencies (markers) at all tensions, Figure 11a,b. As the tension exceeds the value corresponding to $f / f_{c c}=1$ the full model (blue solid line) approaches the behavior of a pre-stressed clamped-clamped beam (red dashed line), as it was shown by calculating the roots in Figure 9. The frequency for the pre-stressed clamped-clamped beam (red dashed line) is obtained by substituting the numerically obtained roots of Eq. (16) into Eq. (17).

For the second mode, Figure 11c,d shows a good agreement, in particular for the long bolt M12x260, Figure 11c. However for the short bolt M12x140 the model only qualitatively captures the nonlinear behavior of the measured frequencies at low tension, Figure 11d. The 
discrepancy in the second mode of the short bolt, Figure 11d, between the experimental results (markers) and the theoretical model (blue solid line), can be explained by the small characteristic wavelength of the second mode, which means that the shear deformation becomes important, and thus the frequency of the second mode obtained from Bernoulli-Euler theory becomes less accurate compared to the first mode. The effective length of each bolt used to produce the blue and the red-dashed lines for the second mode, Figure 11c,d, differs from the one used for the first mode, Figure 11a,b.

Figure 12 shows the eigenvalue ratio of the full model to the pre-stressed clamped-clamped beam versus the bolt tension $N$ for each bolt's first mode. The parameters used to produce Figure 12 are the same as the ones used in Figure 11a,b. As mentioned in Subsection 2.3, Figure 12 shows that the long bolt becomes effectively clamped $\lambda / \lambda_{p c c}=1$ at lower tension than the short bolt. Although the bolt tension in each bolt is the same which leads to a same normal stiffness, the effect of the normal stiffness is different for each bolt due the difference in the characteristic wavelength - the effect of the boundaries stiffness becomes more complex and dominant in the case of short bolt, as the boundaries and the bolt can be seen as one whole elastic body, whereas for the long bolt the boundaries stiffness can be isolated from the bolt and thus considered as true boundaries to the bolt.

The excellent agreement for the first transverse mode shows that the model consisting of a one-dimensional beam, Eqs. (1)-(5), and boundary stiffness, Eq. (28), is useful for explaining the frequency trends of the bolt for the whole range of tension, low and high.

\section{Summary and discussion}

Figure 11 supports the hypothesis that the main cause of the nonlinear squared frequency trend in Figure 4 is the increase in boundary stiffness accompanying increased bolt tension, i.e. bolt tightening. This increase of boundary stiffness with tension in the experiment, which is modeled by Eqs. (27), (28) and (29), is complex for it depends on the geometric and material properties of the mating surfaces. In the nonlinear region $\left(f / f_{c c}<1\right)$ the bolt ends (head/nut) are neither free nor clamped, they are taking different finite values of stiffness that increase as the contact area between the mating surfaces increases, mostly in a nonuniform fashion depending on the asperity distribution pattern in the depth direction and the shape, with the increased pressure. As the pressure, correspondingly the bolt tension, increases, the contact area covers all the mating surfaces and consequently the "infinite" value of the boundary stiffness saturates (cf. Figure 10). Figure 11 also supports the claim that at high tension the pre-stressed bolt behaves as if it is clamped at both ends (head and nut), which results in a linear trend of the squared frequency as the tension is further increased beyond the value corresponding to $f / f_{c c}=1$.

The above results suggest that the bolt first transverse natural frequency can be used as an estimation means to assess in-service bolt tightness. Once the material and geometrical properties of the bolt are known, a simple impact and response measurement on the bolt can give an idea about its loosening/tightening health state. The first transverse natural frequency can also serve to quantify the tension level of an in-service bolt, by using the 


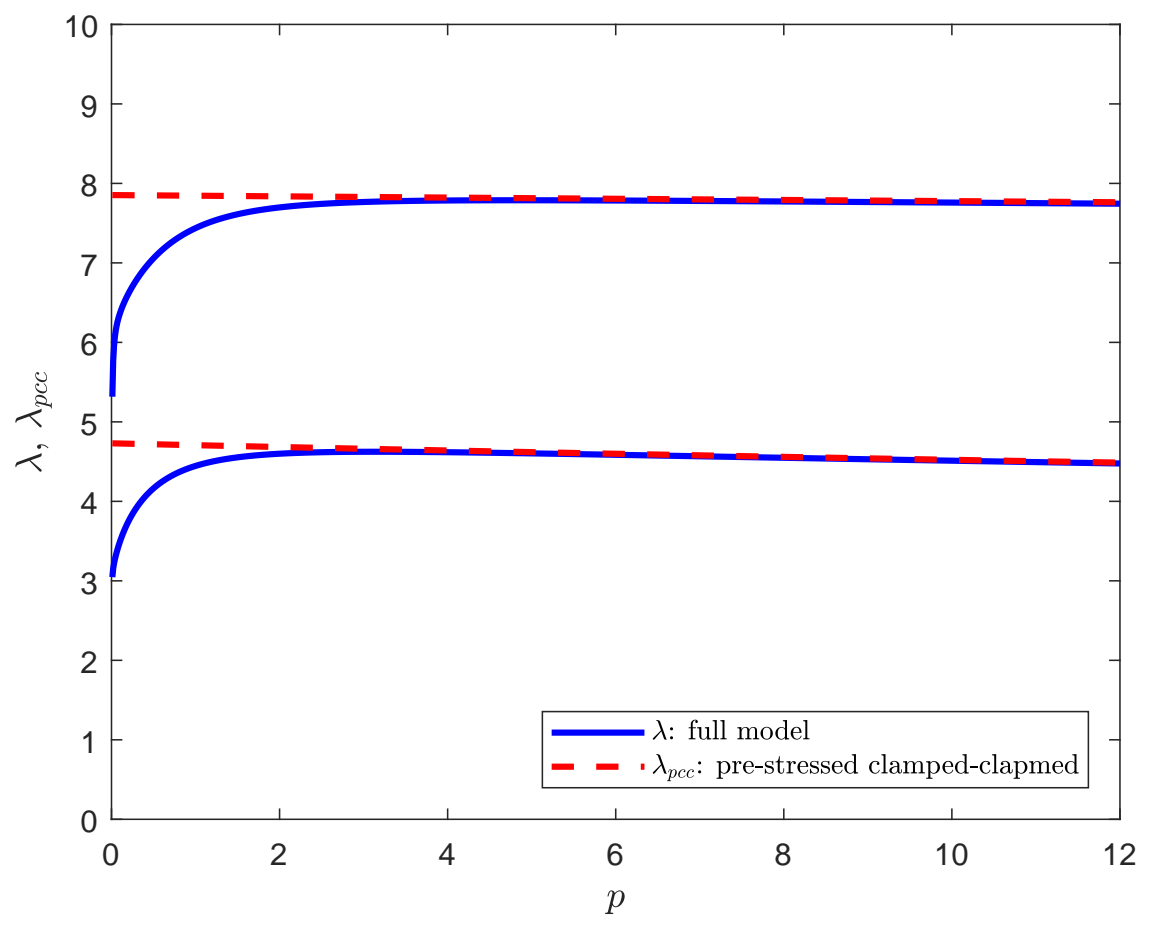

Figure 9: The two lowest roots $\lambda$ (blue solid line) of the frequency equation (30) for finite boundary stiffness, and the roots (red dashed line) of the frequency equation (16) for clamped-clamped boundaries $\lambda_{p c c}$. With parameters $E=207 \mathrm{GPa}, \rho=7850 \mathrm{~kg} / \mathrm{m}^{3}, R=9$ $\mathrm{mm}, r=6 \mathrm{~mm}, \nu=0.3, \cos \theta=1 c_{1}=210^{5}, c_{2}=0.35, c_{3}=20, c_{4}=0.8$; with $l=253.5$ $\mathrm{mm}$ ( $1^{\text {st }}$ mode $)$ and $l=251 \mathrm{~mm}\left(2^{\text {nd }}\right.$ mode $)$.

pre-stressed beam model with the analytical expressions, Eqs. (27), (28) and (29), for the boundary stiffness.

This technique can also be used to estimate bolt tightness during mounting and tightening. A test can be carried out such that three successive tightening loads are applied, then the square of the measured frequency is recorded for each load level, if the three data points form a straight line this means the bolt is well tightened. Otherwise, an extra higher load is applied and the last three data points are checked. This process can be continued until the last three data points form a straight line, indicating the well tightness of the bolt.

\section{Conclusion}

An experiment with two test specimens each clamped with one bolt, has been conducted. Impact modal analysis was performed for the bolt as its tension was increased. The natural frequencies of the two first transverse modes for each bolt was recorded, and results showed 


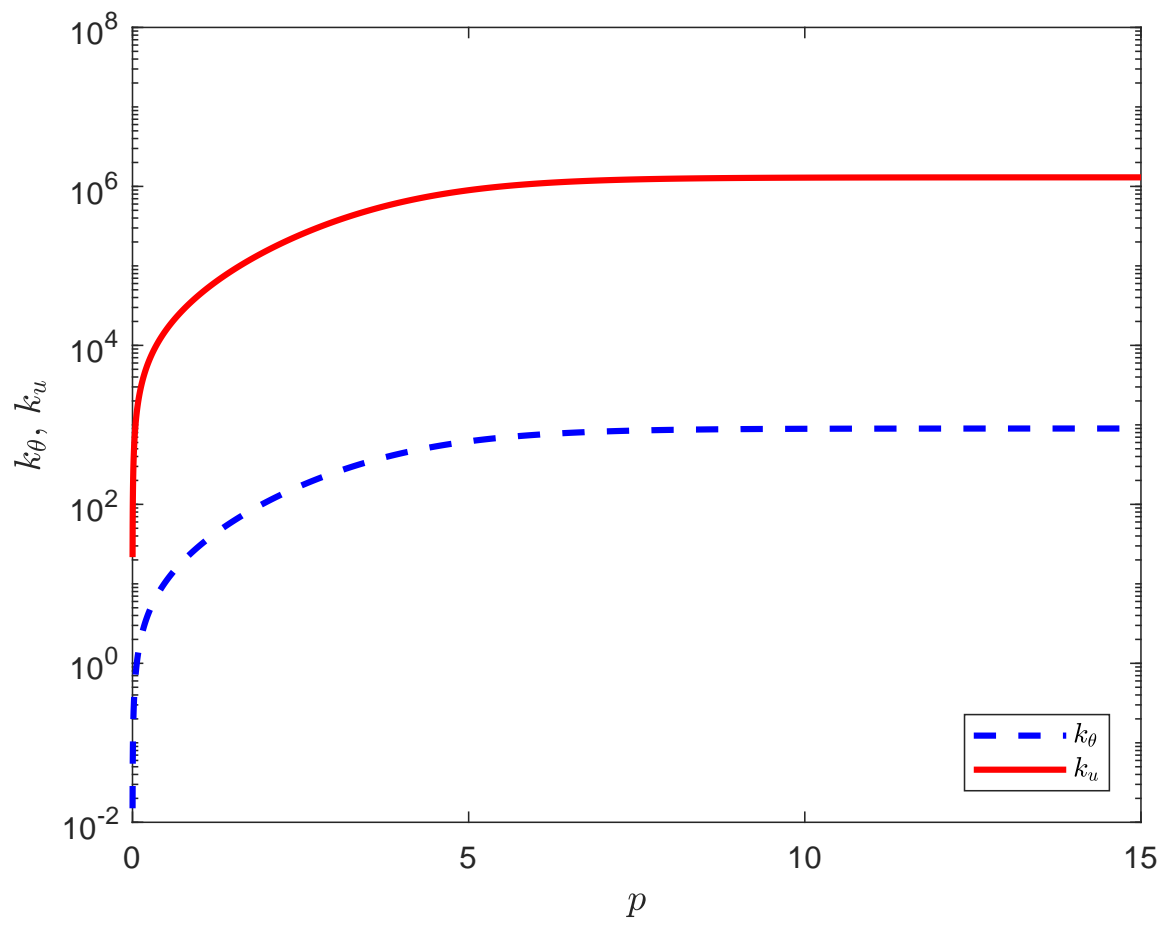

Figure 10: Rotational boundary stiffness (blue dashed line) and translational boundary stiffness (red solid line), Eq. (28), with the same parameters used for the $1^{\text {st }}$ mode in Figure 9 .

that the first mode was more sensitive to the change in bolt tension compared to the second mode. The squared natural frequency trends were shown to exhibit nonlinear and linear behaviors depending on the bolt tension level. A simple model was considered, with an analytical stiffness function proposed to theoretically describe these two behaviors. The analytical results gave an excellent agreement with the measured first mode frequencies, hereby showing that at low tension the nonlinear behavior of the squared frequency versus tension curve can be explained by boundary stiffness depending nonlinearly on tension, and also suggesting that the linear behavior at high tension was due to the bolt being effectively clamped at its both ends (bolt-head/nut).

The sensitivity of the bolt first transverse natural frequency to tension suggests that the squared frequency can be used in assessing the level of bolt tightness. Furthermore, the excellent agreement of the analytical model with the measured data suggests that the bolt tension can be quantified for the whole range of tension, low and high. An extension of this bolt frequency-based technique, would be to test it for a structure with more than one bolt and for a joint when two structures are clamped together with one or more bolts. 

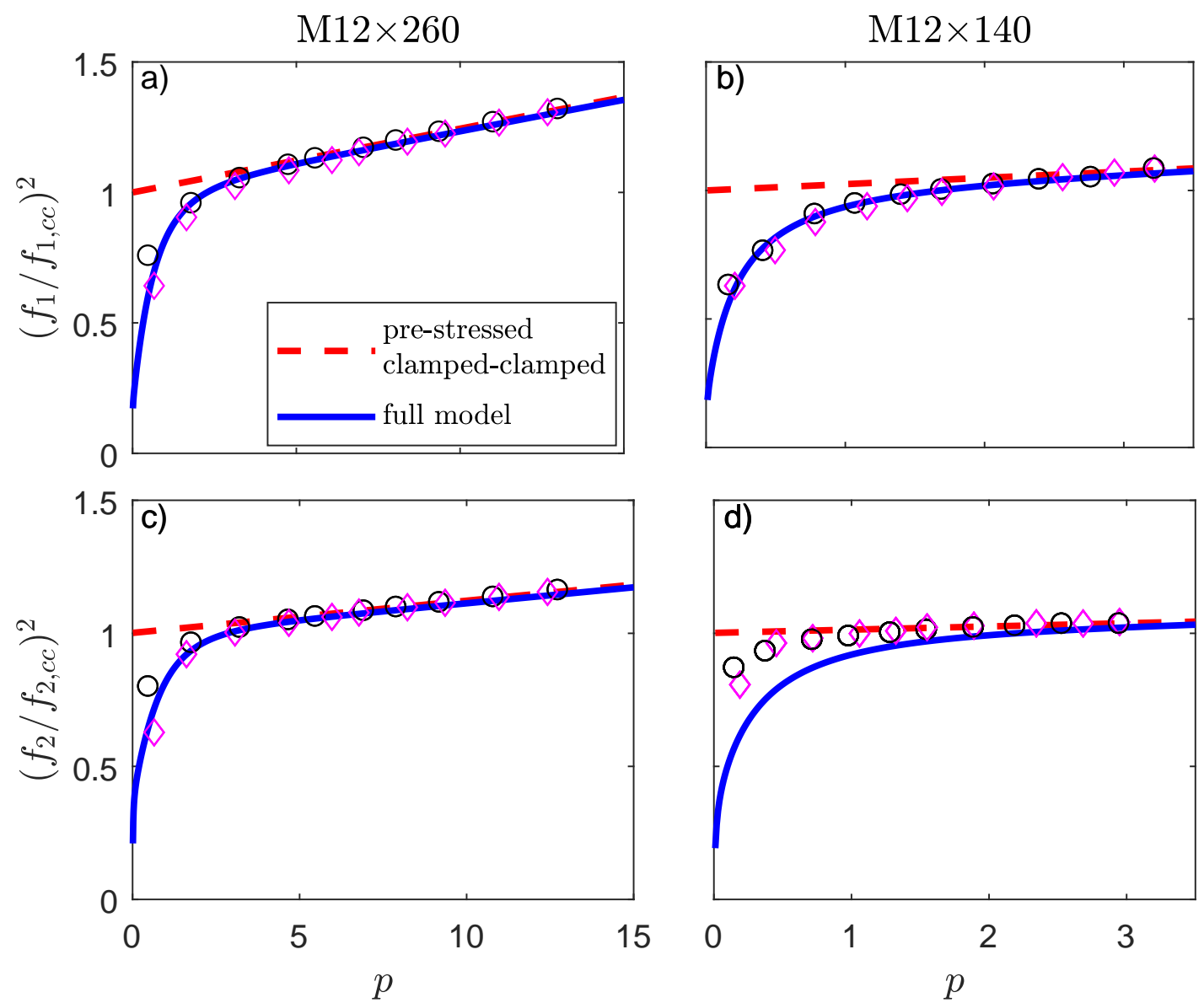

Figure 11: Nondimensional transverse natural frequency of the large bolt M12x260 (a,c) and the short bolt M12x140 (c,d) as function of nondimenisonal tension: Experimental data (markers). Frequency solution to Eq. (30) (blue solid line); frequency solution to Eq. (16) (red dashed line); (a,b) first mode; (c,b) second mode. With parameters: $E=207 \mathrm{GPa}$, $\rho=7850 \mathrm{~kg} / \mathrm{m}^{3}, R=9 \mathrm{~mm}, r=6 \mathrm{~mm}, \nu=0.3, \cos \theta=1$; (a,c) $c_{1}=210^{5}, c_{2}=0.35$, $c_{3}=20, c_{4}=0.8$; (b,d) $c_{1}=210^{5}, c_{2}=0.3, c_{3}=20, c_{4}=0.6$; (a) $l=253.5 \mathrm{~mm}$; (b) $l=127$ $\mathrm{mm}$; (c) $l=251 \mathrm{~mm}$; (d) $l=121 \mathrm{~mm}$.

\section{Acknowledgment}

This work is financially supported by Independent Research Fund Denmark, grant DFF6111-00385. 


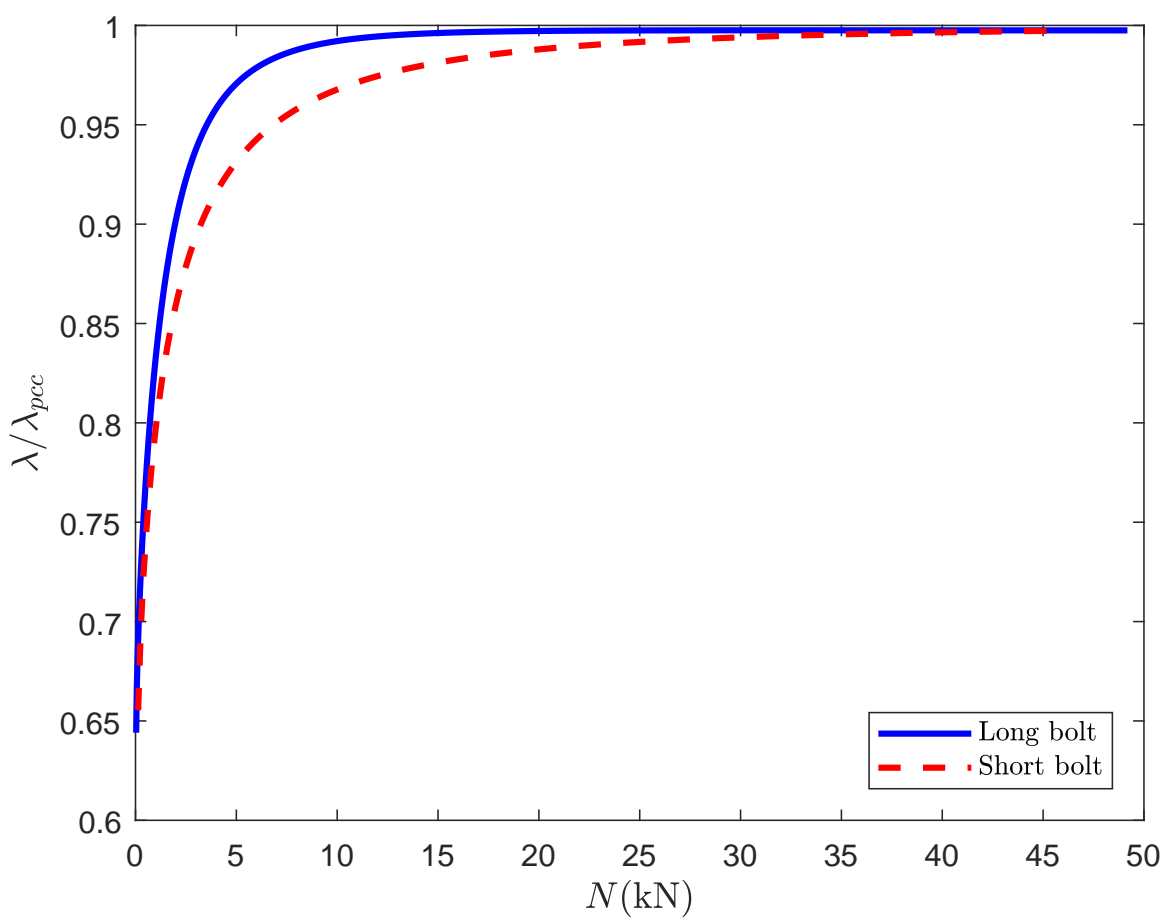

Figure 12: Eigenvalue ratio of the full model Eq. (30) to the pre-stressed clamped-clamped beam Eq. (16) versus bolt tension for the long and short bolt's first mode, with the same parameters used in Figure 11a,b.

\section{References}

[1] D.P. Hess, Vibration- and shock-induced loosening, in: J.H. Bickford, S. Nasser (eds.), Handbook of Bolts and Bolted Joints, Marcel Dekker, New York, 1998.

[2] G. Junker, New criteria for self-loosening of fasteners under vibration, SAE Paper 690055 (1969) 314-335.

[3] J. Bickford, An Introduction to the Design and Behavior of Bolted Joints, Revised and Expanded, Volume 97. CRC press 1995.

[4] T. Fukuoka, T. Takaki, Evaluations of the tightening process of bolted joint with elastic angle control method, American Society of Mechanical Engineers, Pressure Vessels and Piping Conference PVP 4782004.

[5] B. Phares, Y-S. Lee, T. Brockman, J. Rooney, Investigation of High-Strength Bolt-Tightening Verification Techniques, (2016). InTrans Project Reports. 184. https://lib.dr.iastate.edu/intrans_reports/184 
[6] D. Crecraft, The measurement of applied and residual stresses in metals using ultrasonic waves. Journal of Sound and Vibration 5 (1) (1967) 173-192.

[7] S. Joshi, R. Pathare, Ultrasonic instrument for measuring bolt stress, Ultrasonics 22 (6) (1984) 261-269.

[8] S.A. Nassar, A.B. Veeram, Ultrasonic Control of Fastener Tightening Using Varying Wave Speed, Journal of Pressure Vessel Technology 128 (2005) 427432.

[9] H. Yasui, K. Kawashima, Acoustoelastic Measurement of Bolt Axial Load with Velocity Ratio Method: 15th World Conf. on Non-Destructive Testing (Rome, October 1521) pp 750-6, 2000.

[10] S. Chaki, G. Corneloup, I. Lillamand, H. Walaszek, Combination of longitudinal and transverse ultrasonic waves for in situ control of the tightening of bolts, Journal of Pressure Vessel Technology 129(3) (2007) 383-390.

[11] Y. Ito, J.M. Toyoda, S. Nagata, Interface pressure distribution in a bolt-flange assembly, Journal of Mechanical Design 101(2) (1979) 330-337.

[12] M.B. Marshall, R. Lewis, R.S. Dwyer-Joyce, Characterisation of contact pressure distribution in bolted joints, Strain 42 (2006) 3143.

[13] M.B. Marshall, R. Lewis, T. Howard, Ultrasonic measurement of self-loosening in bolted joints, Journal of Mechanical Engineering Science 226 (2012) 18691884.

[14] G. Hartmann, Potentials and Limitations of Ultrasonic Clamp Load Testing, SAE International, Warrendale, PA, 2007-01-1668, 2007.

[15] M.D Todd, J.M Nichols, C.J Nichols, L.N Virgin, An assessment of modal property effectiveness in detecting bolted joint degradation: theory and experiment, Journal of Sound and Vibration 275 (2004) 1113-1126.

[16] J.J. Meyer, D.E. Adams, Theoretical and experimental evidence for using impact modulation to assess bolted joints, Nonlinear Dynamics 81 (2015) 103117.

[17] F. Amerini, M. Meo, Structural health monitoring of bolted joints using linear and nonlinear acoustic/ultrasound methods, Structural Health Monitoring 10(6) (2011) 659672.

[18] A. Milanese, P. Marzocca, J. Nichols, M. Seaver, S. Trickey, Modeling and detection of joint loosening using output-only broad-band vibration data, Structural Health Monitoring 7 (2008) 309328.

[19] T.L. Paez, L.J. Branstetter, D.L. Gregory, Modal randomness induced by boundary conditions, Society of Automotive Engineering Technical Paper (1985) 851930.

[20] E. Oberg, F. Jones, H. Ryffel, C. McCauley, R. Heald, Machinery's Handbook. 29th ed. Industrial Press, p.1610, 2012. 
[21] SKF-Group (2001). Bolt-tightening Handbook (http://kartalrulman.com/UserFiles/katalog/skfkatalog/Mechatronic/Bolt-Tightening/365741185-1.pdf)

[22] J.M. Monville, Optimal tightening process of the bolted joints, International Journal for Simulation and Multidisciplinary Design Optimization 7 (2016) A4.

[23] D.E. Adams, C.R. Farrar, Classifying linear and nonlinear structural damage using frequency domain arx models, Structural Health Monitoring 1(2) (2002) 185201.

[24] D.J. Inman, Engineering Vibration, Prentice Hall, NJ 1996.

[25] J.J. Thomsen, Vibrations and stability: Advanced Theory, Analysis, and Tools, Springer-Verlag Berlin Heidelberg, 2003.

[26] A. Bokaian, Natural frequencies of beams under tensil axial load, Journal of Sound and Vibration 142 (3) (1990) 481-498.

[27] B.W. Drinkwater, R.S. Dwyer-Joyce, P. Cawley, A study of the interaction between ultrasound and a partially contacting solid-solid interface, Proceedings of the Royal Society A 451 (1996) 2613-2628.

[28] J.A. Greenwood, J.B.P. Williamson, Contact of nominally flat surfaces, Proceedings of the Royal Society A 295 (1966) 300-319.

[29] H. Sherif, Parameters affecting contact stiffness of nominally flat surfaces, Wear 145 (1991) 113-121.

[30] H. Sherif, S.S. Kossa, Relationship between normal and tangential contact stiffness of nominally flat surfaces, Wear 151 (1991) 49-62.

[31] R.D. Mindlin, Compliance of elastic bodies in contact, Journal of Applied Mechanics 16 (1949) 259-258.

[32] K.L. Johnson, Contact Mechanics, Cambridge University Press, New York, 1987, p. 464.

[33] K. Kendal, D. Tabor, An ultrasonic study of the area of contact between stationary and sliding surfaces, Proceedings of the Royal Society A 323 (1971) 321-340. 


\section{Appendix A: Nomenclature}

Dimensional variables

$f_{i} \quad i$ th transverse natural frequency the bolt/beam, $\mathrm{Hz}$

$f_{i, c c} \quad i$ th transverse natural frequency of the clamped-clamped beam, $\mathrm{Hz}$

$\tilde{k}_{u} \quad$ translational stiffness at beam ends, $\mathrm{N} / \mathrm{m}$

$\tilde{k}_{\theta} \quad$ rotational stiffness at beam ends, $\mathrm{N}$ m

$N$ tensile axial force (bolt tension, clamping force), $\mathrm{N}$

$\tilde{x}$ longitudinal coordinate, $\mathrm{m}$

$\tilde{t}$ time, s

$\tilde{u}$ beam transverse deflection, $\mathrm{m}$

$l$ length of the beam, $\mathrm{m}$

E Young's modulus, $\mathrm{N} / \mathrm{m}^{2}$

$I$ mass moment of inertia of the cross-section about the neutral axis, $\mathrm{m}^{4}$

EI bending stiffness, $\mathrm{N} \mathrm{m}^{2}$

$\rho \quad$ density of the beam, $\mathrm{kg} / \mathrm{m}^{3}$

$A$ cross-sectional area, $\mathrm{m}^{2}$

$\rho A \quad$ mass per unit length, $\mathrm{kg} / \mathrm{m}$

$w_{0} \quad$ characteristic frequency of the beam, $\mathrm{rad} / \mathrm{s}$

$\eta \quad$ asperity density

$\beta$ asperity radius of curvature, $\mathrm{m}$

$E^{*} \quad$ composite modulus of elasticity of the material of the two surfaces, $\mathrm{N} / \mathrm{m}^{2}$

$\sigma \quad$ composite standard deviation of asperity height distributions, $\mathrm{m}$

$d$ distance between the two surfaces, $m$

$\delta_{n} \quad$ denotes the deformation induced by the force $N, \mathrm{~m}$

$\tilde{k}_{n} \quad$ normal stiffness

$d a$ infinitesimal area of the ring, $\mathrm{m}$

$(\rho, \phi)$ polar coordinates of $\mathrm{d} a$ in the ring surface

$r$ inner radius of the bolt-head/nut, $\mathrm{m}$

$R \quad$ outer radius of the bolt-head/nut, $\mathrm{m}$

$\delta_{\theta} \quad$ deformation induced by tilting of the ring, $\mathrm{m}$

$\tilde{K}_{n} \quad$ normal stiffness density, $\mathrm{N} / \mathrm{m}^{2}$

$M \quad$ total torque about the axis of abscissas, $\mathrm{N} \mathrm{m}$

$\nu \quad$ Poisson ratio 
Dimensionless variables

$$
\begin{aligned}
x & \text { longitudinal coordinate } \\
t & \text { time } \\
u & \text { beam transverse deflection } \\
p & \text { nondimensional tensile axial force } \\
w & \text { beam transverse frequency } \\
\lambda, \gamma & \text { eigenvalues of the full model } \\
\lambda_{c c}, \gamma_{c c} & \text { eigenvalues of the clamped-clamped beam } \\
\lambda_{p c c}, \gamma_{p c c} & \text { eigenvalues of the pre-stressed clamped-clamped beam } \\
h & \text { normalized gap between the two surfaces } \\
\left(c_{1}, c_{2}, c_{3}, c_{4}\right) & \text { fitting parameters for the normal stiffness } \\
c_{\theta} & \text { coefficient for the rotational stiffness } \\
c_{u} & \text { coefficient for the translational stiffness }
\end{aligned}
$$

